\title{
Ist die ästhetische Chirurgie überhaupt noch Medizin? Eine ethische Kritik
}

\author{
Is Aesthetic Surgery Still Really Medicine? An Ethical Critique
}

Autor

Institut
G. Maio

Lehrstuhl für Bioethik, Institut für Ethik und Geschichte der Medizin, Albert-Ludwigs-Universität Freiburg, Freiburg i. Br.

\author{
Schlüsselwörter \\ - ästhetische Chirurgie \\ - Ethik \\ - Jugendwahn \\ - Konsumgesellschaft \\ - Eitelkeit \\ - Beauty-Industrie \\ - Identität der Medizin \\ Key words \\ - aesthetic surgery \\ - ethics \\ - bioethics \\ - youth mania \\ - vanity \\ - consumer society \\ - beauty industry \\ - identity of medicine
}

eingereicht 16.4.2007

akzeptiert 27.4.2007

\section{Bibliografie}

DOI 10.1055/s-2007-965328 Handchir Mikrochir Plast Chir 2007; 39: 189-194 @ Georg Thieme Verlag KG Stuttgart . New York • ISSN 0722-1819

\section{Korrespondenzadresse}

Prof. Dr. med. Giovanni Maio Lehrstuhl für Bioethik

Institut für Ethik und Geschichte der Medizin

Albert-Ludwigs-Universität

Freiburg

Stefan-Meier-Straße 26

79104 Freiburg i. Br.

giovanni.maio@uniklinik-

freiburg.de

\section{Zusammenfassung \\ $\nabla$}

In den letzten Jahren versteht sich die ästhetische Chirurgie zunehmend als ein Dienstleistungsgewerbe, das die Wünsche ihrer Klienten zum Ausgangspunkt ästhetischer Interventionen macht. In ethischer Hinsicht stellt sich die Frage, ob es überhaupt gerechtfertigt sein kann, dass eine medizinische Sparte sich vom medizinischen Kernauftrag der Leidenslinderung verabschiedet und stattdessen ein reiner Dienstleistungsanbieter auf Wunsch wird. Im Beitrag werden die Argumente beleuchtet, die gegen eine unreflektierte Anbindung und Anbiederung der Medizin an die Beauty-Industrie sprechen. So läuft eine rein ökonomisch orientierte ästhetische Chirurgie Gefahr, Wünsche zu erfüllen und zugleich zu wecken, die in sich deswegen problematisch sein können, weil sie von der Ideologie der Jugendlichkeit und der Machbarkeit getragen werden. Vor allem macht sich eine solche „Chirurgie“ die Ideologien einer Leistungs- und Konsumgesellschaft zu nutze, um daraus Profit zu schlagen. Als Schlussfolgerung wird dafür plädiert, dass jeder ästhetische Chirurg in aufrichtiger Weise darum wirbt, den Wert des Menschen nicht auf seine äußere Erscheinung zu reduzieren. So lange die ästhetische Chirurgie durch ihre Marktorientierung diese aufrichtige Aufklärungsarbeit vernachlässigt, wird sie sich als genuin ärztliche Institution mittelfristig abschaffen.

Weit entfernt scheint die Zeit, als die Medizin noch von Patienten angerufen wurde, von leidenden Menschen, die in Not waren und sich vertrauensvoll an ihren Arzt wandten, damit dieser sein Können in den Dienst des leidenden Mitmenschen stellte, ohne Ansehen der Person, ohne Ansehen der wirtschaftlichen Möglichkeiten des Patienten und ohne Eigeninteresse des Arztes. Weit entfernt scheint diese Zeit, denn blickt man

\section{Abstract \\ $\nabla$}

Aesthetic surgery has evolved in the past years from a genuine medical practice to a mere commodity. From an ethical point of view one must ask whether this evolution creates more problems than it solves. The present paper elaborates four arguments against this evolution and shows that an aesthetic surgery which works only according to market categories runs the risk of losing the view for the real need of patients. An aesthetic surgery that understands itself as part of a market will be nothing else than a part of a beauty industry which has the only aim to sell something, but not the aim to help people. Such an aesthetic surgery makes profit from the ideology of a society that serves only vanity, youthfulness and personal success and which is losing the sight for real values. The real value of man cannot be reduced to its appearance and medicine as an art should feel the obligation to resist these modern ideologies and should help people to get a more authentic attitude to themselves. If aesthetic surgery fails to think about these implications it will lose its identity as medicine which would be a too great loss.

auf die heutige Dienstleistungsmedizin, so drängt sich der Eindruck auf, dass heute nicht mehr der leidende Patient seinen Arzt aufsucht, sondern dass die Medizin selbst mit ihren Angeboten ihre nicht leidenden Nichtpatienten umwirbt, um ihre eigenen (Lifestyle-)Produkte abzusetzen. Eine solche Entwicklung der Medizin wird sich in Zukunft noch weiter verstärken, weil viele Kliniken und Praxen gerade vor dem Hinter- 
grund ökonomischer Engpässe zusätzliche Einnahmequellen in der Dienstleistungsmedizin sehen werden, die nicht selten eine existenzsichernde Basis darstellen. $\mathrm{Zu}$ den gängigsten Produkten, für die die Medizin Abnehmer sucht, gehören „medizinische“ Maßnahmen zur „Verbesserung“ des äußeren Erscheinungsbildes des Menschen. Diese Produkte sind zum Beispiel in den Sparten der sogenannten Anti-Aging-Medizin anzutreffen [7], sie werden aber auch von vielen Zahnärzten [8], von Dermatologen und nicht zuletzt auch von ästhetischen Chirurgen in immer größerer Zahl angeboten und auf Wunsch angewendet. Viele Ärztinnen und Ärzte halten einen solchen Umbau der Medizin nicht nur für vollkommen unbedenklich, sondern immer mehr setzen sich für eine solche Ausrichtung der Medizin sogar werbend ein. Dieser Trend wirft einige ethische Fragen auf, die im Folgenden mit besonderem Schwerpunkt auf die Identität der Medizin vertieft werden sollen.

\section{Die ästhetische Chirurgie als marktgeleitete Disziplin \\ $\nabla$}

Was motiviert ästhetische Chirurgen, den Körper nach den Wünschen der Klienten zu modellieren? Natürlich gibt es einige Körperformen, die - trotz des natürlichen Vorkommens - für manche Menschen so entstellend wirken, dass beispielsweise eine extrem auffällige Nase einen beträchtlichen Leidensdruck erzeugen kann. Ein solcher Leidensdruck könnte eine hinreichende Rechtfertigung dafür sein, dass eine natürliche Körperform chirurgisch verändert wird. Das Ziel einer solchen Maßnahme wäre die Linderung eines Leidens. Auch wenn kritisch angemerkt werden muss, dass das Leiden nicht aus der Körperform selbst resultiert, sondern aus der Deutung dieser Körperform durch eine ästhetisch vorgeprägte Mediengesellschaft, so kann in einem solchen Falle dennoch der positive Nutzen einer chirurgischen Intervention für das Selbstwertgefühl und die soziale Integration des Betroffenen so groß sein, dass es ethisch gerechtfertigt erschiene, chirurgisch tätig zu werden.

Wie sieht es aber nun aus, wenn Menschen den ästhetischen Chirurgen mit der Bitte aufsuchen, dass er ihren Körper nach einem bestimmten Medienidol forme oder wenn Menschen ihren Körper modelliert haben wollen, weil sie sich dadurch einen Wettbewerbsvorteil sowohl in der Partnerwahl als auch im beruflichen Leben versprechen? Was wäre in diesem Fall die Motivation des Chirurgen, wenn er dem Anliegen nachkäme? Ein Leidenszustand liegt in diesen Fällen nicht vor, sodass der Chirurg hier nicht mehr Leidenslinderer wäre. Daraus wird schon deutlich, dass die Grundlage der ästhetischen Chirurgie in vielen Fällen nicht die Erfüllung eines medizinischen Bedarfs, sondern allein die Erfüllung eines Wunsches ist. Hyman hat die grundlegende Moral der ästhetischen Chirurgie wie folgt treffend beschrieben: „When you are happy, I'm happy“ [3]. Der ästhetische Chirurg ist somit in vielen Fällen kein Heiler, sondern lediglich Wunscherfüller. Das Spezifische an der reinen Wunscherfüllung liegt nun genau darin, dass für die Auswahl der entsprechenden Klienten nur zwei Parameter von Relevanz sind: der Wunsch an sich und die finanzielle Kaufkraft des Klienten. Anders formuliert: Für eine solche ästhetische Chirurgie sind allein der Geschmack und der Geldbeutel des Klienten ausschlaggebend. Das Ziel eines solchen ästhetischen Chirurgen ist dann nicht mehr die Linderung von Leid, sondern die Gewinnoptimierung des „Unternehmens Ästhetische Chirurgie“. Mit einem solchen Ziel verabschiedet sich die „Medizin“ von ihrem ureigenen Auftrag, ein Ort der Hilfe zu sein.

Diese Umorientierung hat weitreichende Folgen. So besteht die Gefahr, dass ab dem Moment, da die Chirurgie als Leitmotiv ihres Handelns tatsächlich nur noch den Markt, die Gewinnoptimierung und die persönlichen und oft narzisstischen Wünsche ihrer „Kunden“ definitiv wählte, sie alsbald in eine Identität zurückfallen könnte, die sie eher in die Nähe des Barbiers als in die Nähe der ärztlichen Identität (zurück-)brächte.

Das Arztsein ist eine Profession und nicht nur ein Beruf. Mit dem Status der Profession sind Privilegien verbunden, die darin bestehen, dass der Staat sich weitgehend bei der Formulierung der Standards für die Ausübung der Profession heraushält. Der Staat vertraut darauf, dass die Ärzte selbst festlegen, was eine gute Medizin ist. Das Privileg der Medizin als Profession liegt darin, dass sie von sich aus sagen kann, wie eine Behandlung nach den Regeln der ärztlichen Kunst auszusehen hat. Der Staat verlässt sich darauf, weil er davon ausgeht, dass die Medizin als Medizin sich von einem moralischen Wert, nämlich dem Wohl des Patienten - und nur von diesem - in ihrer Festlegung der Standards leiten lässt. Ähnlich beruht auch das Vertrauen des Patienten weniger in der technischen Versiertheit des Arztes als darin zu wissen, dass wenn er Arzt ist, er als Arzt sicher zu allererst an seinen Patienten denken wird. Von einem Verkäufer wird man eine solche moralische Grundeinstellung nicht erwarten, und jeder weiß, dass der Verkäufer zunächst einmal daran denkt, sein Produkt zu verkaufen. Ein Arzt aber kann als Arzt nicht zuerst an den Verkauf denken, sondern er muss zuerst daran denken, ob seine Leistung tatsächlich gut für den Patienten ist. Genau hierin liegt der vulnerable Punkt der heutigen ästhetischen Chirurgie, wenn sie sich als marktgesteuerte Dienstleistungsmedizin organisiert.

Wenn die Medizin den Verkauf ihrer Dienstleistungen gegen Geld an autonome Kunden (wie autonom sind sie wirklich?) zu ihrem eigentlichen Ziel erklärt, so ist das von sich aus nicht zu verurteilen, aber es muss klar sein, dass mit einer solchen modern klingenden Ausrichtung eine wegweisende Grundsatzentscheidung getroffen wird. Ab dem Moment, da der Verkauf im Vordergrund steht, ab diesem Moment taucht die Frage auf, ob hier überhaupt noch von Medizin die Rede sein kann. Wenn das Leitmotiv das Verkaufen ist und nicht das Helfen, dann ist es kein ärztliches Handeln mehr, sondern Handeln eines Dienstleistenden. Am Ende eines solchen Trends stünde also die Ablösung einer moralischen Institution durch ein moralindifferentes Handwerk. Folge eines solchen Identitätswandels wäre es, dass ein reiner Dienstleistender nicht mehr die Privilegien in Anspruch nehmen dürfte, die der Arzt als Teil einer Profession zu Recht in Anspruch nimmt. Das heißt - zu Ende gedacht - dass ein Arzt, der etwas verkaufen möchte und hierbei betont, dass er Arzt ist, unmoralisch handelt. Denn er benutzt den Nimbus, der mit dem Arztsein verknüpft ist, nur für seine eigenen Interessen, obwohl er streng genommen gar nicht als Arzt, sondern nur als Dienstleistender handelt. Er müsste also, um moralisch integer zu handeln, zugeben, dass er hier nicht als Arzt, sondern lediglich als Dienstleistender - vielleicht sogar als Handwerker handelt. Der Dienstleistende, der Handwerker ist nur für das Resultat verantwortlich, er bringt sein technisches Können ein, ohne hinterfragen zu brauchen, ob das Ziel, wofür er es einbringt, ein gutes Ziel ist. Für den Arzt kann dieses Ziel nur das Wohl seines Patienten sein. Eine rein marktökonomisch ausgerichtete ästhetische Medizin wird dieses Wohl nur noch als Nebeneffekt im Auge haben, jedoch nicht mehr als primäres Ziel. Daher wäre 
eine rein sich dem Markt verschreibende ästhetische Chirurgie eine defizitäre Chirurgie, ja sie wäre keine Medizin mehr.

\section{Die ästhetische Chirurgie als eine dem Patientenwohl ferne Disziplin}

Natürlich ließe sich einwenden, dass die ästhetische Chirurgie doch gar nicht den Markt, sondern nach wie vor das Wohl des „Patienten“ zum Ausgangspunkt macht. Man könnte einwenden, dass die chirurgisch hergestellte Faltenfreiheit zum Wohlbefinden des „Patienten“ beitragen würde. Da die Weltgesundheitsorganisation die Gesundheit gleichgesetzt hat mit physischem, psychischem und sozialem Wohlbefinden, erscheint es auf den ersten Blick gerechtfertigt, unter Bezugnahme auf diese Definition auch die ästhetische Chirurgie als „Medizin“ zu betrachten, da sie der so verstandenen „Gesundheit“ dient. Doch hier gilt es $\mathrm{zu}$ bedenken, dass diese WHO-Definition alles andere als eine vernünftige Grundlage für die Definition von Medizin sein kann. Denn wenn das psychische Wohlbefinden Ziel der Medizin sein soll, dann wäre beispielsweise auch die Finanzberatung eine medizinische Maßnahme, da diese gerade bei überschuldeten Menschen tatsächlich zu einem größeren „Wohlbefinden“ beitrüge und das Leid eines überschuldeten Menschen lindern könnte. An diesem Beispiel lässt sich unschwer erkennen, dass das „Wohlbefinden“ allein nicht ausreicht, um eine Maßnahme als eine medizinische zu bezeichnen. Vor allem macht das Beispiel deutlich, dass eine Subsumierung solcher Maßnahmen unter die Rubrik Medizin dazu führen würde, dass alle Bereiche des Lebens medikalisiert werden würden. Zwar hat die Medizin als Markt ein Interesse daran, alle Lebensbereiche zum Zuständigkeitsbereich der Medizin zu erklären - man denke nur an die gesamte "Anti-Aging-Medizin“ - aber von der Sache her ist eine solche Ausweitung nicht gerechtfertigt und trägt dann auch dazu bei, dass normale Zustände illegitimerweise pathologisiert werden. Genau dies geschieht auch landläufig, wenn die ästhetische Chirurgie sich als Medizin ausgibt und mit dem Skalpell Probleme zu lösen vorgibt, die eigentlich keine medizinischen sind. Denn - so muss kritisch nachgefragt werden - was ist das Grundproblem, das hinter dem Ansinnen eines Menschen steht, so auszusehen wie ein bestimmtes Popidol? Oder was ist das Grundproblem, das einen Menschen dazu veranlasst, bei einem normalen Körpergewicht dennoch Fettareale wegoperieren zu lassen? Oder was ist das Grundproblem, wenn Männer sich ihren Unterkiefer „auspolstern“ lassen, um nicht nur männlicher, sondern auch durchsetzungsfähiger auszusehen? Mit dem Skalpell wird in diesen Fällen kein medizinisches Problem „behandelt“, sondern eindeutig ein soziales. Ein soziales deswegen, weil die Grundursache für die beschriebenen „Wünsche“ an die Medizin die Verfasstheit unserer Leistungsgesellschaft ist und nicht das Aussehen selbst.

Freilich lässt sich einwenden, dass die Gesellschaft nicht leicht zu ändern ist, aber dann stellt sich die schwerwiegende Frage, ob denn ein soziales Problem tatsächlich durch den chirurgischen Eingriff gelöst wird oder ob nicht eher eine Scheinlösung vorliegt. Eine Scheinlösung deswegen, weil ab dem Moment, da normal aussehende Menschen mit ihrem Aussehen nicht zufrieden sind, diese Menschen eher eine Stärkung ihres Selbstwertgefühls bräuchten als die Veränderung ihrer Körperformen. Daher wäre ein ästhetischer Chirurg dann ein Arzt, wenn er seinem Klienten genau dies sagen würde, dass es eine effektivere und vor allem langfristiger anhaltende Behandlungsmöglichkeit gäbe, nämlich die Arbeit an der eigenen Persönlichkeit. Wer ein gemindertes Selbstbewusstsein mit dem Skalpell zu lösen glaubt und vor allem zu lösen vorgibt, wird nicht ernsthaft am Wohl seines Patienten interessiert sein, sondern eher an der Bilanz seines Haushaltes. Da in diesem Fall also die ökonomische Zielsetzung über der Zielsetzung des Patientenwohls steht, ist hier keine echte Hilfe gegeben, sondern lediglich ein Geschäft vollzogen, und zwar ausgehend von einem Dienstleistenden, dem das Wohl des Verbrauchers mehr oder weniger gleichgültig ist.

Dass das Patientenwohl nicht zwangsläufig das herrschende Kriterium der ästhetischen Chirurgie ist, lässt sich an folgendem banal erscheinenden Beispiel verdeutlichen:

Eine Frau kommt zum Arzt wegen einer Routineuntersuchung, und während der Routineuntersuchung weist der Arzt sie darauf hin, dass die moderne Chirurgie mittlerweile in der Lage sei, ohne besondere Nebenwirkung auch Hakennasen zu begradigen. Die Frau schaut den Arzt verdutzt an und beteuert, dass sie mit ihrer Nase bisher immer zufrieden gewesen sei. Der Arzt lächelt nur müde und schweigt. Auf dem Nachhauseweg fallen der Frau längst vergessene Bilder wieder ein: wie sie als kleines Mädchen ob der Hakennase gehänselt worden war, und im selben Augenblick fällt ihr ein, dass eine entfernte Bekannte sich auch einer solchen Operation unterzogen hat. Am Schluss zweifelt sie, ob der Arzt nicht vielleicht doch irgendwie Recht gehabt haben könnte.

In diesem Falle hat der Arzt gerade nicht ärztlich gehandelt, weil er erst eine Nachfrage geschaffen hat, die ohne ihn nicht aufgekommen wäre. Und nach diesem Prinzip funktioniert auch der Markt. Wenn die ästhetische Chirurgie ihre Kunden nur auf diese Weise rekrutierte, wäre sie nicht als Medizin zu beschreiben, denn in diesem Falle sorgt der Arzt nicht für eine Leidenslinderung, sondern er sorgt erst für das Entstehen eines Leidenszustandes, für den er erst sekundär eine „Hilfe“ bereitstellt. Implizit handelt es sich in diesem Falle um nichts anderes als um eine versteckte Werbung für ein medizinisches Produkt. Denn die Werbung macht es sich zum Ziel, einen Bedarf zu wecken; das heißt, dass der Arzt mit seiner Werbung nichts anderes bezweckt als dem Menschen das Gefühl zu geben, dass es ihm ohne kosmetischen Eingriff eigentlich nicht gut gehen kann. Die Werbung sorgt also dafür, dass Menschen, die sich vor der Werbung wohl fühlen, durch die Werbung dieses Wohlempfinden verlieren, damit es durch die Maßnahme des Arztes wieder hergestellt werde. Hieraus wird deutlich, in welch schwerwiegender Weise die Werbung den ärztlichen Auftrag zur Hilfe konterkariert. Wäre das Wohlergehen des Patienten der eigentliche oder gar einzige Beweggrund und die eigentliche Zielsetzung ärztlichen Handelns, so wäre die Werbung kein geeignetes Mittel, um dieses Ziel zu erreichen. Die Werbung ist somit für den Umworbenen schlecht; sie ist aber gerade für die Medizin schlecht, denn ab dem Moment, da der Arzt Werbung für bestimmte kosmetische Maßnahmen betreibt, wird implizit zum Ausdruck gebracht, dass das primäre Ziel dieses ärztlichen Handelns das Verkaufen oder der Gewinn eines Marktwettbewerbs ist und nicht das Wohl des Patienten.

Dass das werbende Angebot gerade von einem Arzt kommt, ist sehr bedeutsam für die soziale Wahrnehmung dieses Angebots. Wenn nun ein Arzt eine ästhetische Operation durchführt, so erfährt diese Operation allein aufgrund des Status Arzt eine andere Bedeutung als wenn ein Nichtarzt etwas tut, denn implizit gehen viele Menschen davon aus, dass wenn ein Arzt eine solche Operation durchführt, sie in irgend einer Weise vernünftig und 
indiziert sein muss, denn sonst würde sie der Arzt doch nicht vornehmen. In unserem konkreten Fall könnte die beunruhigte Frau denken, dass wenn es der Arzt schon empfiehlt, es ja gar nicht so abwegig sein kann, sich einer solchen Operation zu unterziehen. Ab dem Moment, da der Arzt eine bestimmte Nase operiert, sendet er gleichsam ein Signal in die Gesellschaft, und zwar das Signal, dass eine solche Nase nicht akzeptabel ist. Mag der einzelne Chirurg dies persönlich gar nicht so empfinden, aber allein die Tatsache, dass ein Arzt eine solche Operation durchführt, suggeriert, dass die Maßnahme in irgendeiner Weise indiziert ist. Dies hängt damit zusammen, dass medizinische Eingriffe im Allgemeinen als invasiv und risikoreich wahrgenommen werden, und ab dem Moment, da der Eingriff vorgenommen wird, wird implizit davon ausgegangen, dass das Nutzen-Risiko-Verhältnis ausgewogen ist [5].

All dies hat zur Folge, dass die Institutionalisierung der ästhetischen Chirurgie unweigerlich Normen setzt. In einer Kultur, in der der Körper nach ästhetischen Gesichtspunkten modelliert wird, wird am Ende auch der durchschnittliche Körper als mangelhaft empfunden werden. Wenn das Ideal zur Norm gemacht wird, dann ist die Abweichung vom Ideal bereits „Krankheit“. Die ästhetische Chirurgie sorgt also allein durch ihre Angebote und Maßnahmen dafür, dass viele sich allein dadurch defizitärer fühlen als sie es täten, wenn es keine ästhetische Chirurgie gäbe. Denn die Frage, welche Nase, welche Hüfte, welche Brust veränderungsbedürftig ist und welche nicht, ist im Grunde keine Frage der persönlichen Präferenzen des „Patienten“, sondern letztlich eine Frage der gesellschaftlichen Konvention, und an der Entstehung dieser Konvention trägt die Medizin wesentlich bei. Dass die Medizin hier einen Einfluss auf die gesellschaftliche Wahrnehmung des normalen Körpers haben kann, sei an einem chirurgiefernen Beispiel, nämlich am Beispiel der Zahnspange erläutert:

Vor einer Generation war es ganz normal, mehr oder weniger schiefe Zähne zu haben. Mittlerweile wird ein sehr hoher Prozentsatz an Schulkindern mit einer Spange versehen. Diese Ausweitung der Indikation zur Spange führt dazu, dass - obwohl schiefe Zähne von der Natur eher die Norm als die Ausnahme sind - mittlerweile nicht der natürliche Zustand des mehr oder weniger schiefen Gebisses, sondern der Idealzustand des komplett gleichmäßigen Gebisses zur Norm geworden ist. Derjenige, der keine Spange hatte, wird - obwohl der natürliche Zustand belassen - zunehmend zum Außenseiter. Bei der Spange ist die Indikation zwar keine rein ästhetische, aber dieses Beispiel macht deutlich, in welchem Wechselverhältnis gesellschaftliche Erwartungen an ein bestimmtes Aussehen auch und gerade durch das medizinische Angebot verstärkt werden können [5]. Schlussfolgernd stellt sich die schwerwiegende Frage, ob die ästhetische Chirurgie - als reine Marktwirtschaft verstanden - allein durch ihr Angebot nicht ihren Auftrag als helfende Disziplin gerade dadurch verfehlt, weil sie keinen echten Beitrag für das Wohl des Patienten darstellt. Schwerwiegender noch muss gefragt werden, ob die ästhetische Chirurgie nicht vielmehr einen Schaden bewirkt, gerade weil die Verbreitung ästhetischer Eingriffe in vielen Menschen den Eindruck verstärkt, dass ihr Erscheinungsbild sozial nicht akzeptabel ist. Indem die Medizin an der Realisierung der Schönheitsideale mitwirkt, entwickelt sie eine nicht nur normenrezeptive, also eine reagierende Funktion, sondern die Medizin hat dann auch eine normensetzende Kraft. Sie bestätigt mit ihrem Tun nicht zuletzt auch Stereotypien und Vorurteile, und sie bekräftigt Modeströmungen. Für die Menschen, die sich durch die Modeströmungen verunsichert fühlen, weil sie Angst haben, der Jugendlichkeitsmode nicht entsprechen zu können, für diese Menschen ist die ästhetische Chirurgie durch deren Bestätigung der Trends eher ein Schaden als ein Nutzen.

\section{Die ästhetische Chirurgie als Erfüllungsgehilfin einer fragwïrdigen Konsumgesellschaft $\nabla$}

Die letzten Überlegungen führen uns zu einer weiteren zentralen Kritik an der ästhetischen Chirurgie. Ein „Arzt“, der allein den Wunsch des Patienten als Legitimation seines „ärztlichen“ Tuns begreift, ohne den Wunsch selbst noch einmal kritisch zu reflektieren, läuft Gefahr, bestimmte Vorstellungen zu bestätigen, die für sich genommen kritikwürdig sind. So müssen wir zum Beispiel fragen: Was ist das für eine Vorstellung vom Menschen, wenn man davon ausgeht, dass man nur mit dieser oder jener Erscheinung ein lebenswertes Leben führen kann? Was ist das für ein Menschenbild, wenn man die persönliche Anerkennung von der Form einer Hüfte oder von der Brustgröße abhängig macht? So gilt es zu bedenken, dass beispielsweise die antike Philosophie die Schönheit immer nur in der Verknüpfung mit der Tugend definiert hat. Schönheit und Tugend waren in dieser antiken Tradition lange Zeit als zwei Seiten einer Einheit betrachtet worden; ohne das eine sei das andere nicht möglich, wo das eine ist, liegt auch das andere nahe [4]. Heute hat man die Schönheit reduziert auf das rein Äußere und hat sie der Tugend, der inneren Haltung komplett entrissen. Folge hiervon ist es, dass die Suche nach einer solchen Schönheit nunmehr nicht mehr die Suche nach einer ganzheitlichen Einheit ist. Vielmehr ist die heutige Suche nach Schönheit Ausdruck einer wahnhaften Rastlosigkeit einer Gesellschaft, die nicht eine ganzheitliche Einheit im Sein sucht, sondern sich mit dem richtigen Schein begnügt.

Eine Medizin, die auf breiter Front rein kosmetisch handelt, wird am Ende mitverantwortlich sein für eine Gesellschaft, die dem Primat der Eitelkeit, der Äußerlichkeit, der Jugendlichkeit und der sinnentleerten Oberflächlichkeit folgt. Die Medizin mag nicht der Urheber dieser Ausrichtung sein, aber indem sie sich offiziell - und meist ohne Skrupel - die Ästhetik zu einer ihrer zentralen Aufgaben macht, bestätigt und fördert eine solche Medizin diese latent vorhandenen modernen Tendenzen.

Die Fixierung auf Äußerlichkeiten, die Hochschätzung des reinen Scheins und die Reduzierung des Menschen auf seine Leistungsfähigkeit und seine rein äußerliche „Attraktivität“ sind ein Bestandteil der beklagenswerten Verstrickung der modernen Medizin. Doch darüber hinaus trägt die moderne Medizin auch dazu bei, dass der postmoderne Mensch sich immer mehr als ein Mensch versteht, der selbst darüber entscheiden kann, wer und was er ist [10]. Das heißt, dass die ästhetische Chirurgie zu einer in der Medizin wie in der modernen Gesellschaft weit verbreiteten Machbarkeitsideologie beiträgt. Der Mensch lehnt in dieser modernen Ideologie seine eigenen natürlichen Grenzen ab; er begreift sich selbst nicht mehr als gegeben, sondern nur als gemacht, und dementsprechend ist er dem Wahn verfallen, auch seinen Körper immer weiter zu „machen“. Der Blick dafür, dass der Mensch in den Kernbereichen seines Lebens nichts machen, sondern nur in Gelassenheit erwarten und vertrauen kann, dieser Blick ist dem modernen Menschen abhanden gekommen. Die Medizin sorgt mit ihren marktgesteuerten Ansätzen dafür, dass der moderne Mensch sich immer mehr der Einsicht versperrt, dass jeder Mensch als Mensch ein endliches und grund- 
sätzlich begrenztes und unvollkommenes Wesen ist, das nur glücklich werden kann in der Annahme seiner Unvollkommenheit. Daher ist eine zentrale Kritik an der ästhetischen Chirurgie gerade darin zu sehen, dass eine solche Medizin sich zur Komplizin einer Gesellschaft macht, die einem unreflektierten Machbarkeitswahn unterliegt. Eine solche Medizin hat sich dafür entschieden, keine Institution der Hilfe mehr zu sein, sondern nur noch Erfüllungsgehilfin einer rastlosen Gesellschaft, die so etwas wie Sinn nur noch in der wahnhaften Selbstgestaltung erblickt [6]. Denn dass das Äußere für so viele postmoderne Menschen einen so hohen Stellenwert einnimmt, liegt gerade daran, dass viele Menschen in einem Sinndefizit leben, den sie mit solchen Äußerlichkeiten zu kompensieren versuchen. Nehmen wir die für die ästhetische Chirurgie so relevante Einstellung zum Älterwerden.

\section{Die ästhetische Chirurgie als eine altersfeindliche Disziplin \\ $\checkmark$}

Viele Menschen suchen den ästhetischen Chirurgen auf, damit dieser ihnen helfe, die physiologischen (!) Alterserscheinungen zu kaschieren. Hierfür hat die Disziplin ästhetische Chirurgie ein breites Arsenal an wirksamen Methoden entwickelt, die in allen Handbüchern aufgeführt werden. Damit, dass viele ästhetische Chirurgen alle Falten gegen Geld wegoperieren, vermitteln sie den Eindruck, dass ein gutes Leben nur dann geführt werden könne, wenn man nicht altersentsprechend, sondern so jung wie technisch möglich aussieht. Damit bestätigt und bekräftigt die ästhetische Chirurgie die Schönheitsideale unserer Gesellschaft, die insofern problematisch sind, als diese eine Glorifizierung der Jugendlichkeit und zugleich eine Herabsetzung des alten Menschen implizieren. So ist die Tatsache, dass die Medizin das Altsein mit technischen Mitteln zu verbergen versucht, und dass sie hierfür Mittel nicht nur anwendet, sondern auch anpreist, alles andere als trivial. Denn damit suggeriert die Medizin, dass das Altaussehen - und damit das Altsein - ein bedauernswürdiger Zustand ist, der behoben werden müsste. Mit der ästhetischen Medizin verstärkt die Medizin die modernen Tendenzen, das Altsein als etwas Defizitäres zu betrachten. Eine so pejorisierende Betrachtung des Altseins ist zwar kein Spezifikum des 21.Jahrhunderts. Vielmehr hat es seit der Antike immer wieder solche Assoziationen gegeben [2]. Die Medizin macht sich den wiederbelebten Trend zur Kultivierung der Jugendlichkeit in einer exzessiven Weise zunutze und schlägt daraus Profit. Da dieser Trend als solcher kritikwürdig ist und auf Kosten der Alten und Schwachen geht, ist das Profitschlagen in doppelter Hinsicht problematisch. Nicht nur, weil das Profitschlagen per se ein Problem der Medizin als Disziplin der Hilfe ist, sondern die Grundlage für dieses Profitschlagen ist problematisch, weil der Profit nur durch eine fragwürdige und kritikwürdige Vorstellung vom Menschsein möglich geworden ist.

Kritikwürdig ist eine solche Pejorisierung des Alters vor allem in anthropologisch-ethischer Hinsicht [9]. Indem die Medizin mehr oder weniger unreflektiert Mittel anpreist, durch die die Älteren nach jugendlichen Vorbildern äußerlich umgestaltet werden sollen, macht sich die Medizin zur Komplizin einer Gesellschaft, die im Altsein nur noch eine Schwundstufe des Menschen sieht. Die Medizin sorgt auf diese Weise mit dafür, dass das Altsein nur dann akzeptiert wird, wenn der Mensch auch im Altsein noch jugendlich schön ist. Versteht man die Medizin als eine Institution der Hilfe, so könnte man ihr im Umgang mit dem alternden Menschen eine andere helfende Aufgabe zuschreiben als jene, den alternden Menschen dabei zu helfen, das Altsein und die spezifischen Merkmale des Altwerdens zu ignorieren und zu verdrängen. Indem ältere Menschen von der Medizin äußerlich jugendlich gemacht werden, werden diese alten Menschen gerade nicht in ihrem Altsein respektiert, sondern nur insofern sie noch so geblieben sind wie die Menschen in der mittleren Lebensphase. Mit allen Mitteln nicht alt aussehen wollen ist somit Ausdruck einer Ideologisierung der mittleren Lebensphase. Eine solche Selbstdeutung des Menschen ist in ethischer Hinsicht mehr als problematisch, weil sie den Wert und den Sinn des Altseins ignoriert.

Die ästhetische Medizin möchte aus Altgewordenen wieder Junggebliebene machen. Als Institution der Hilfe könnte die Medizin sich auch alternativ dafür einsetzen, einen gesunden Umgang mit dem Altwerden zu unterstützen. Sie könnte alternativ zur unreflektierten Wunscherfüllung den Altgewordenen aufzeigen, dass man auch und gerade in seiner äußeren wie inneren Gebrechlichkeit ein lebenswertes Leben führen kann, sofern man es schafft, sich von den Ideologien der Jugendlichkeit und Leistungsorientiertheit der mittleren Lebensphase zu lösen. Als Institution der Hilfe könnte die Medizin alternativ zur ästhetischen Chirurgie um die Einsicht werben, dass das Gute nicht allein durch das gute Machen und Gestalten verwirklicht werden kann, sondern dass gerade im Altern und nicht nur da das Gute auch durch ein Sein zur Realisierung kommen kann. Kaum eine andere Domäne unserer Gesellschaft nimmt einen so starken Einfluss auf unsere Bilder vom Altsein wie die Medizin. Daher ist die Medizin gut beraten, über ihr Tun gerade im Zuge eines zunehmenden Ästhetikbooms kritischer nachzudenken.

\section{Wie kann ein Arzt trotzdem helfen? \\ $\nabla$}

Welche konkrete Konsequenz ergibt sich aus all dem Gesagten für die ethische Bewertung der ästhetischen Chirurgie? Die vorangegangenen Überlegungen sollen deutlich machen, dass die ästhetische Chirurgie in Zukunft ihre Glaubwürdigkeit und ihre Identität als ärztliche Disziplin nur dann bewahren kann, wenn sie verantwortungsbewusst mit der Indikationsstellung umgeht. Ein Arzt, der nur reflexartig das tut, was der Klient von ihm erwartet, läuft Gefahr, vom Klienten zum reinen Erfüllungsgehilfen degradiert zu werden. Ein solcher Arzt könnte genauso gut ein Handwerker sein. Was den Arzt zum Arzt macht, ist gerade die kritische Reflexion des Patientenwunsches, ist die Implementierung von Verantwortung. Konkret bedeutet das, dass der gute Arzt den Wunsch des Patienten nach einem ästhetischen Eingriff stets kritisch reflektiert. Er operiert nicht automatisch, sondern erst wenn er sich sicher ist, dass der Wunsch des Patienten nicht auf Realitätsverluste, auf übersteigerte Phantasien oder auf problematische anthropologische Vorverständnisse zurückzuführen ist.

In diesen Fällen kommt gerade dem klärenden Gespräch eine ganz besondere Bedeutung zu; im Zweifelsfalle werden bestimmte Eingriffe erst nach weiterer Abklärung vorgenommen. In manchen Fällen müssten Klientenwünsche auch abgelehnt werden. Zwar würde eine solche Ablehnung den Umsatz verringern, aber eine solche Haltung wäre dafür eine Investition in die Zukunft.

Wenn sich der ästhetische Chirurg als Arzt verstünde, müsste er nicht nur vermeiden vorzugeben, Probleme zu lösen, die mit dem Skalpell gerade nicht zu lösen sind. Er müsste darüber 
hinaus andere Schönheitsideale propagieren, die nicht einseitig sind und die die Schönheit gerade nicht auf die äußere Form reduzieren, sondern die sie als Gesamteindruck eines Menschen begreift. Der Philosoph Gernot Böhme hat Schönheit nicht als Prädikat, sondern als eine Weise zu sein definiert [1]. In dieser Konzeption wird Schönheit nicht erst durch den Chirurgen möglich, sondern nur durch die Arbeit an einer anderen Ausstrahlung, an einem anderen Auftreten, an einer anderen Kommunikationsweise, an einer anderen Einstellung zu sich und der Welt. Daher wäre eine Aufgabe gerade des ästhetischen Chirurgen, für eine Schönheit zu plädieren, die gerade nicht darin besteht, alle Menschen durch das Skalpell zu uniformieren, sondern die darin bestünde, gerade die Diversität menschlichen Aussehens positiv zu konnotieren. Mit einer solchen Überzeugungs-, ja Aufklärungsarbeit würde der Chirurg seinem Patienten in vielen Fällen einen größeren Dienst erweisen als mit einem invasiven Eingriff. Ferner müsste der Chirurg für folgende Einsicht werben: Es mag sein, dass durch ein „besseres“ Aussehen so mancher Wettbewerbsvorteil verknüpft sein könnte, aber dieser Wettbewerbsvorteil - sei er privat oder beruflich - hat zwei Fallstricke. So muss gefragt werden: was sind das für Menschen, die allein einer Äußerlichkeit wegen sich positiv einstellen lassen? In der Regel wird es hier lediglich um eine kurzfristige und sehr oberflächliche Bevorzugung gehen. Ferner müsste auch hier bedacht werden, dass das Bevorzugtwerden sich ja nicht nur durch das Aussehen, sondern in gleicher Weise durch eine andere Ausstrahlung und durch ein soziales Auftreten erreichen ließe. Wenn es der Arzt ernst meint mit der Hilfe für den Patienten, so würde er doch vielmehr darin investieren, den Patienten dabei zu helfen, mehr soziale Kompetenz zu erwerben, weil diese die gleichen „Wettbewerbsvorteile“ mit sich brächte und darüber hinaus für den Patienten viel billiger zu bekommen wäre. Der ästhetische Chirurg, der - als Arzt - in einer Weise „hilft“, die viele Risiken in sich birgt, teuer für den Patienten ist und zugleich ineffektiver ist als andere Formen, soziale oder private Anerkennung zu erlangen, ein solcher Chirurg wird seinem Auftrag als Arzt jedenfalls nicht gerecht. Daher wird die ästhetische Chirurgie als Medizin nur dann eine Zukunft haben können, wenn sie das zurückgewinnt, was sie am meisten verloren hat, nämlich das Vertrauen in ihre moralische Integrität.

\section{Hinweis}

$\nabla$

Nach einem Vortrag anlässlich der Jahrestagung der DGPRÄC in Aachen vom 29. September bis 2. Oktober 2006.

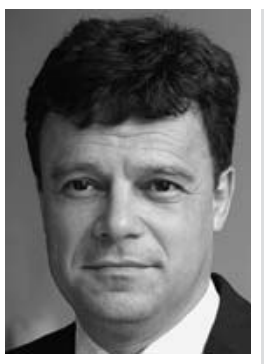

Giovanni Maio

Geb. 1964. Studium der Medizin und Philosophie in Freiburg, Straßburg und Hagen. 1990 bis 1995 Facharztweiterbildung Innere Medizin, 2000 Habilitation für Ethik und Geschichte der Medizin in Lübeck. 2002 Berufung in die Zentrale Ethikkommission für Stammzellenforschung durch die Bundesregierung. 2004 Rufe auf C4-Professuren in Aachen, Bochum und Zürich. Seit 2005 Inhaber des Lehrstuhls für Bioethik an der Medizinischen Fakultät der Universität Freiburg. Seit 2006 Direktor des Instituts für Ethik und Geschichte der Medizin und Geschäftsführender Direktor des Interdisziplinären Ethik-Zentrums Freiburg.

\section{Literatur}

1 Böhme G: Leibsein als Aufgabe. Leibphilosophie in pragmatischer Hinsicht. Kusterdingen: Die Graue Edition, 2003

2 Engelhardt D: Altern zwischen Natur und Kultur. Kulturgeschichte des Alters. In: Borscheid P (Hrsg): Alter und Gesellschaft. Stuttgart: Hirzel, 1995: $13-23$

3 Hyman DA: Aesthetics and ethics: the implications of cosmetic surgery. Perspect Biol Med 1990; 33: 190-202

4 Maio G: Is etiquette relevant to medical ethics? Ethics and aesthetics in the works of John Gregory. Med Health Care Philos 1999; 2: 181 187

5 Maio G: Die ästhetische Chirurgie und das ärztliche Selbstverständnis - eine medizinethische Betrachtung. In: Wolff H, Welzel J, Engelhardt D (Hrsg): Ethik in der Dermatovenerologie. Lübeck: Hansisches Verlagskontor, 2002: 139-147

6 Maio G: Das Menschenbild als Grundfrage der Medizinischen Ethik. In: Blum H, Haas R (Hrsg): Über das Menschenbild in der Medizin. Stuttgart: Thieme, 2004: 41-47

7 Maio G: Die Präferenzorientierung der modernen Medizin als ethisches Problem. Ein Aufriss, am Beispiel der Anti-Aging-Medizin. Z Med Ethik 2006; 52: 339-354

8 Maio G: Ethische Grenzen kosmetischer Maßnahmen in der Zahnheilkunde. Zahnarztl Mitt 2006; 96: 78-83

9 Rentsch T: Altern als Werden zu sich selbst. In: Borscheid P (Hrsg): Alter und Gesellschaft. Marburger Forum Philippinum. Stuttgart: Hirzel, 1995: $53-62$

10 Schmidt K, Bayertz K: „Es ist ziemlich teuer, authentisch zu sein...!” Von der ästhetischen Umgestaltung des menschlichen Körpers und der Integrität der menschlichen Natur. In: Ach JS, Pollmann A (Hrsg): No body is perfect. Baumaßnahmen am menschlichen Körper. Bioethische und ästhetische Aufrisse. Bielefeld: transcript Verlag, 2006: $43-62$ 